\title{
Students' Awareness about Psychosocial Problems Associated with Cyber Bullying
}

\author{
Ali Rezk El Khateeb ${ }^{\mathbf{1}}$, Mona Hassan Abdelal ${ }^{2}$ and Naglaa Fathi El-attar ${ }^{\mathbf{3}}$ \\ (1) Registered Nurse at Ministry of Health at Hosh Isa Critical Care Unit (2) Professor of \\ Psychiatric \& Mental Health Nursing- Faculty of Nursing-British University - Egypt and (3) \\ Assistant Professor of Psychiatric \& Mental Health Nursing- Faculty of Nursing- Benha \\ University- Egypt.
}

\section{Abstract}

Background: Cyber bullying is the new power equation attached to bullying issue. In the meantime, digital technology has changed the way adolescents communicate and learn. It affects their behavior and attitude. Aim of the study: Was to assess students' level of awareness about psychosocial problems associated with cyber bullying. Research design: A descriptive exploratory design was used in this study. Setting: The study was conducted in two faculties at Benha University (Nursing and Education). Subjects: A sample of 400 students, 200 from each faculty were chosen by a purposive sample. Tools: Three tools were used for data collection. Tool (1): Structured interview questionnaire was used to collect data about students and family characteristics. Tool (2): Cyber bullying questionnaire was used to assess level of awareness of students about cyber bullying. Tool (3): Psychosocial problems questionnaire was used to assess awareness of students about psychosocial problems associated with cyber bullying. Results: Majority of nursing students had unsatisfactory knowledge about cyber bullying, while more than three quarter had unsatisfactory knowledge about cyber bullying for education students. Three quarters of nursing students have severe psychological effect of cyber bullying meanwhile, less than two thirds of education students have severe psychological effect of cyber bullying. Conclusion: There was lack of awareness of students about psychosocial problems associated with cyber bullying. Recommendations: Psycho-educational training program to improve awareness of students about cyber bullying causes, effects and how to prevent should be conducted.

Key words: Cyber bullying, Psychosocial problems, Students' awareness.

\section{Introduction:}

One of the most important characteristics of the new society is the increasing effect of online communication methods, especially the Internet, on people; there is no wonder that these effects is larger on adolescents than other humans in society. Social media attract all ages. In spite of the many advantages offered by social media and social networks as providing a tool to communicate with friends, opportunities for professional online networking, there are growing concerns of cyber bullying, online harassment, internet use and addiction related to social media behavior (Raheem\& AlKhaldi, 2021).

New social media such as Facebook, YouTube, Instagram, and Twitter are webbased and app-based sites that enable people to interact with each other by sharing texts, photos, opinions and events. These social media have transformed the manner of life and business by reaching other people instantly. Many harmful effects of social media are also reported along with the good uses of these media. One of the most harmful usages of these media is cyber bullying where 
these media is used to annoy, intimidate, or humiliate another online user. These media are being used by some users to post harmful or annoying data and pictures, to write aggressive or rough comments about others, and to share videos proposed to prejudice or embarrass other people (Kumari \& Singh, 2021).

Cyber bullying is a form of bullying that made using new technology such as cellphones, hardware systems, and remote tablets. Cyber bullying can happen offline in social media, platforms, or while playing games where people could see, take part in, or share data with one another. Sending, receiving, posting, or sharing bad, harmful, false or mean content about someone is considered cyber bullying. It may include degrading or annoying someone by sharing personal or private data about them. Some cases of cyber bullying may be considered illegal or criminal (Kumar, 2021).

$$
\text { Cyber bullying found to have }
$$
continuous harmful effects on the victim, causing stress, putting them in a constant state of upset or anxiety leading to sleep disorder and appetite problems. In contrary with physical bully, the cyber bullying posts can be shared with others all around the world with a click to mouse or finger tap, making the victim more fearful and stressed. The perpetrator in cyber bullying may remain anonymous, which increase the problem and make it critical. The victims of cyber bullying were founded to feel jumpy, nervous, sad, and in some cases commit suicide (Kumari \& Singh, 2021).

Nursing role in cyber bullying management first addressing cyber bullying in clinical settings. Then discussion of cyber bullying effects, causes and how to deal with it. Be aware of symptoms of cyber bullying. Screen cyber bullying in clinical settings. Provide support and resources for cyber bulling victims. Enhance policies to control cyber bullying and to prevent cyber bullying before its happening (Megan\& Vaillancourt, 2017).

\section{Significance of the study}

According to new studies the percentages of individuals who have experienced cyber bullying at some point during their lifetime has doubled, with $18 \%$ in 2007 and $36 \%$ in 2019, and this is only expected to continue raising taking the high use of IT, social networks and mobile devices by adolescents and teenagers into account. Based on that it is important to search about cyber bulling causes, ways and preventions (Lopez-Vizcaino, et al., 2021).

In Egypt, it found that, among more than 600 elementary school students, $21.7 \%$ reported bullying perpetration, $28.6 \%$ reported victimization and $9.5 \%$ were bully/victims (Zych et al., 2017).

Cyber bullying is associated with emotional and psychosocial problems and can lead to suicidal ideation. It is also associated with internalizing and externalizing problems, as well as depression, stress and anxiety, and alterations in the cortisol release pattern. Cyber bullying also has an impact on general aspects of subjective well-being such as satisfaction with life or happiness. So it's very important to assess the level of students' awareness about psychosocial associated with cyber bullying.

\section{Aim of the study}

The aim was to assess level of students' awareness toward psychosocial problems associated with cyber bullying.

\section{Research question}

What is the level of students' awareness about psychosocial problems associated with cyber bullying? 


\section{Subject and Methods}

\section{Research design}

A descriptive exploratory design was used in this study.

\section{Setting}

The study was carried out at faculty of Nursing Benha University. It consists of four grades. The students' numbers of first grade (572), the second are (442), the third grades are (461), the fourth grades are (453) and the total number of students is (1928).

Another sample was taken from Faculty of Education Benha University. It consists of four grades. The students numbers of first grade (2140), the second are (2249), the third (2212), the fourth (2233) and the total is (8834).

\section{Sample}

It was a purposive sample. The sample size had been calculated using the following equation: $n=(z 2 \times p \times q) / D 2$. The average sample size at power $80 \%$ and CI $95 \%$ ranged from (371) so that the sample was (400) student, (200) from each faculty.

\section{Tools for data collection}

Three tools were used in this study.

Tool I: Structured interview questionnaire it was developed by the investigator it was divided into two parts:

Part one: Socio- demographic Data: to elicit data about students characteristics such as age, sex, faculty, year of study, number of sisters and brothers, work, income, father and mother education, work and family income.

Part two: Family characteristics data: to elicit data about students family characteristics such as father's education and occupation, mother's education and occupation, number of siblings, birth order and family income.

Tool two: Cyber bullying questionnaire:

It was developed by investigator under guidance of supervisors to assess knowledge of students about cyber bullying, it was about 19 questions.

Tool three: Psychosocial problems questionnaire: it was developed by investigator under guidance of supervisors to assess awareness of students about psychosocial problems associated with cyber bullying. It was consists of two parts.

Part one: Psychological effect questionnaire: Contain 25 closed ended questions to assess students' awareness about cyber bullying psychological effect. It was answered with yes or no

Part two: Social effect questionnaire: Contain 11 closed ended questions to assess students' awareness about social effect of cyber bullying. It was answered with yes or no.

\section{Scoring system}

\begin{tabular}{|c|c|c|c|c|}
\hline Items & $\mathrm{N}$ & Range & Score & \\
\hline \multirow{2}{*}{$\begin{array}{l}\text { Awareness } \\
\text { of students }\end{array}$} & \multirow[t]{2}{*}{19} & $\begin{array}{l}\text { Less } \\
\text { than } \\
75 \%\end{array}$ & $0-14$ & Unsatisfactory \\
\hline & & $\begin{array}{l}75 \text { and } \\
\text { more }\end{array}$ & $\begin{array}{l}15- \\
19\end{array}$ & Satisfactory \\
\hline \multirow{3}{*}{$\begin{array}{l}\text { Psychologi } \\
\text { cal Effect }\end{array}$} & \multirow{3}{*}{25} & $50 \%$ & $0-12$ & Mild \\
\hline & & $\begin{array}{l}>50 \%- \\
75 \%\end{array}$ & $\begin{array}{c}13- \\
19\end{array}$ & Moderate \\
\hline & & $>75 \%$ & $\begin{array}{l}20- \\
25 \\
\end{array}$ & Severe \\
\hline \multirow{3}{*}{$\begin{array}{l}\text { Social } \\
\text { effects }\end{array}$} & \multirow{3}{*}{11} & $50 \%$ & $0-5$ & Mild \\
\hline & & $\begin{array}{l}>50 \%- \\
75 \%\end{array}$ & 6-8 & Moderate \\
\hline & & $>75 \%$ & $9-11$ & Severe \\
\hline
\end{tabular}

\section{Ethical considerations:}

The objective and aim of the study were clarified by the investigator to every participant in the study, oral consent obtained from each student before conducting the interview and they were assured for maintaining anonymity and confidentiality. The students were informed that they have the right to participate in the study and the right to withdraw from the study at any time. 


\section{$\underline{\text { Ali Rezk El Khateeb, Mona Hassan Abdelal and Naglaa Fathi El-attar }}$}

\section{Content validity of the tools}

Arabic translation was done by investigator for tools. Content validity of tools was done by jury of 5 expertise (4 in Psychiatric Nursing Field from Benha, Tanta and Ain-shams University and 1 in Psychiatric Medicine Field from Benha) who checked the relevancy, comprehensiveness, clarity and applicability of the questions. According to their opinions modifications were done and the final form was developed.

\section{Reliability of the tools}

Reliability of tools was tested by using Cronbach's alpha coefficient test, which revealed that the tools consisted of relatively homogenous items as showed by the moderate to high reliability of each tool. Cronbach's alpha of first tool was (0.71) and (0.92) for second tool and (0.83) for third tool.

\section{A pilot study}

It was carried out on $30 \%$ students (120 students) under study to test the applicability, clarity and efficiency of the tools. No modification was done, so the students who shared in the pilot study were included in the sample.

\section{Field work}

- The investigator started data collection by introducing himself to the students.

- Brief description about the purpose of the study and the type of questionnaire required to fill was given to each student.

- Data collection was done by interviewing each student individually or group of students.

- The investigator started data collection from 8 am to $3 \mathrm{pm}$ with interviewing students in the garden of each faculty.

- The interview lasted from 10 -15 mints with each student depending on the response of them.
All data collected were organized, coded, computerized, tabulated and analyzed by using The Statistical Package for Social Science (SPSS programs version 20), which used frequencies and percentages for qualitative descriptive data, Chi-square was used for relation tests, mean and standard deviation was used for quantitative data and person correlation coefficient (r) was used for correlation analysis and degree of significance was identified. A highly statistical significant difference was considered if $\mathrm{p}$-value $<0.01$, statistical significant difference was considered if $\mathrm{p}$ value $<0.05$ and non-statistical significant difference was considered if $\mathrm{p}$-value $\mathrm{p}>$ 0.05 .

\section{Results}

Table (1): Reveals that more than half and less than two thirds of the studied students aged between (18-21) years old in nursing $(52 \%)$ and in faculty of education $(65 \%)$. In contrast, more than two thirds $(67.5 \%)$ are females at faculty of nursing and less than half $(49 \%)$ are females at education faculty. Also, more than three quarters are single (79\% and $80.5 \%$ respectively). Studied students are equal (200) in each group. Regarding studying year, more than one third (37.5\%) of nursing students at fourth year and more than one third (41\%) of education students at second year.

Figure (1): Clarifies that majority of nursing students $(88 \%)$ had unsatisfactory knowledge about cyber bulling but, while more than three quarters $(77 \%)$ had unsatisfactory knowledge about cyber bulling for education students.

Figure (2): Illustrates that three quarters of nursing students have severe psychological effect of cyber bullying meanwhile, less than 


\section{Students' Awareness about Psychosocial Problems Associated with Cyber Bullying}

two thirds of education students have severe psychological effect of cyber bullying.

Figure (3): Presents that majority of nursing students $(88 \%)$ have severe social effects of cyber bullying compared to more than three quarters $(77 \%)$ have severe social effects for education students.

Table (2): Shows that there is highly statistically significant relation between sociodemographic characteristics and knowledge about cyber bullying among students at faculty of nursing. Also, there is statistically significant relation between age, marital status, studying year and knowledge about cyber bullying among students at faculty of education. While, there is no relation between student sex at faculty of education \& knowledge about cyber bullying.

Table (3): Demonstrates that there is highly statistically significant relation between studying level and psychological effects of cyber bullying among nursing students. Adding to that, there is highly statistically significant relation between sex, marital status, grade and psychological effects of cyber bullying among education students.
Table (4): Clarifies that there is highly statistically significant relation between sex, marital status, studying level and social effects of cyber bullying among nursing students. In addition, there is highly statistically significant relation between sex, studying level among education students, while there is no relation with age.

Table (5): Reveals that there is negative correlation between knowledge of cyber bullying and psychological effects. Also, there is negative correlation between knowledge about cyber bullying and social effects. In contrast, there is positive correlation between psychological and social effects of cyber bullying among students of faculty of education.

Table (6): Reveals that there is negative correlation between knowledge of cyber bullying and psychological effects. Also, there is negative correlation between knowledge of cyber bullying and social effects. In contrast, there is positive correlation between psychological and social effects of cyber bullying among nursing students. 
Table (1): Frequency and percentage distribution of sociodemographic characteristics of the studied students $(\mathrm{N}=400)$.

\begin{tabular}{|c|c|c|c|c|}
\hline \multirow{2}{*}{$\begin{array}{c}\text { Socio demographic } \\
\text { Characteristics }\end{array}$} & \multicolumn{2}{|c|}{ Nursing students $\mathrm{N}=200$} & \multicolumn{2}{|c|}{ Education students $\mathrm{N}=200$} \\
\hline & $\mathrm{N}$ & $\%$ & $\mathrm{~N}$ & $\%$ \\
\hline \multicolumn{5}{|l|}{ Age } \\
\hline $18<21$ & 104 & 52.0 & 130 & $65.0 \%$ \\
\hline $21<24$ & 96 & 48.0 & 70 & $35.0 \%$ \\
\hline Mean \pm SD & \multicolumn{2}{|c|}{$21.03 \pm 1.77$} & \multicolumn{2}{|c|}{$20.55 \pm 0.99$} \\
\hline \multicolumn{5}{|l|}{$\overline{\text { Sex }}$} \\
\hline Male & 65 & 32.5 & 102 & $51.0 \%$ \\
\hline Female & 135 & 67.5 & 98 & $49.0 \%$ \\
\hline \multicolumn{5}{|l|}{ Marital status } \\
\hline Married & 42 & 21.0 & 39 & $19.5 \%$ \\
\hline Single & 158 & 79.0 & 161 & $80.5 \%$ \\
\hline \multicolumn{5}{|l|}{ Grade } \\
\hline First & 24 & 12.0 & 46 & $23.0 \%$ \\
\hline Second & 29 & 14.5 & 82 & $41.0 \%$ \\
\hline Third & 72 & 36.0 & 22 & $11.0 \%$ \\
\hline Fourth & 75 & 37.5 & 50 & $25.0 \%$ \\
\hline
\end{tabular}

\section{Total knowledge about cyberbullying}

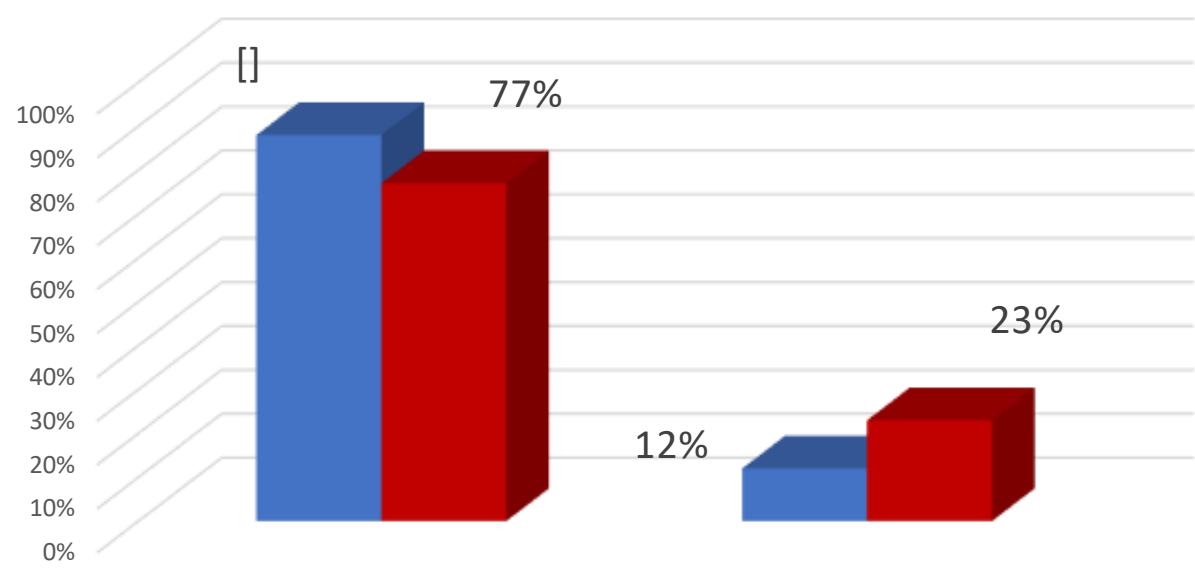

Unsatisfactory Satisfactory

Nursing Education

Figure (1): Percentage distribution of total knowledge about cyber bullying among studied students 


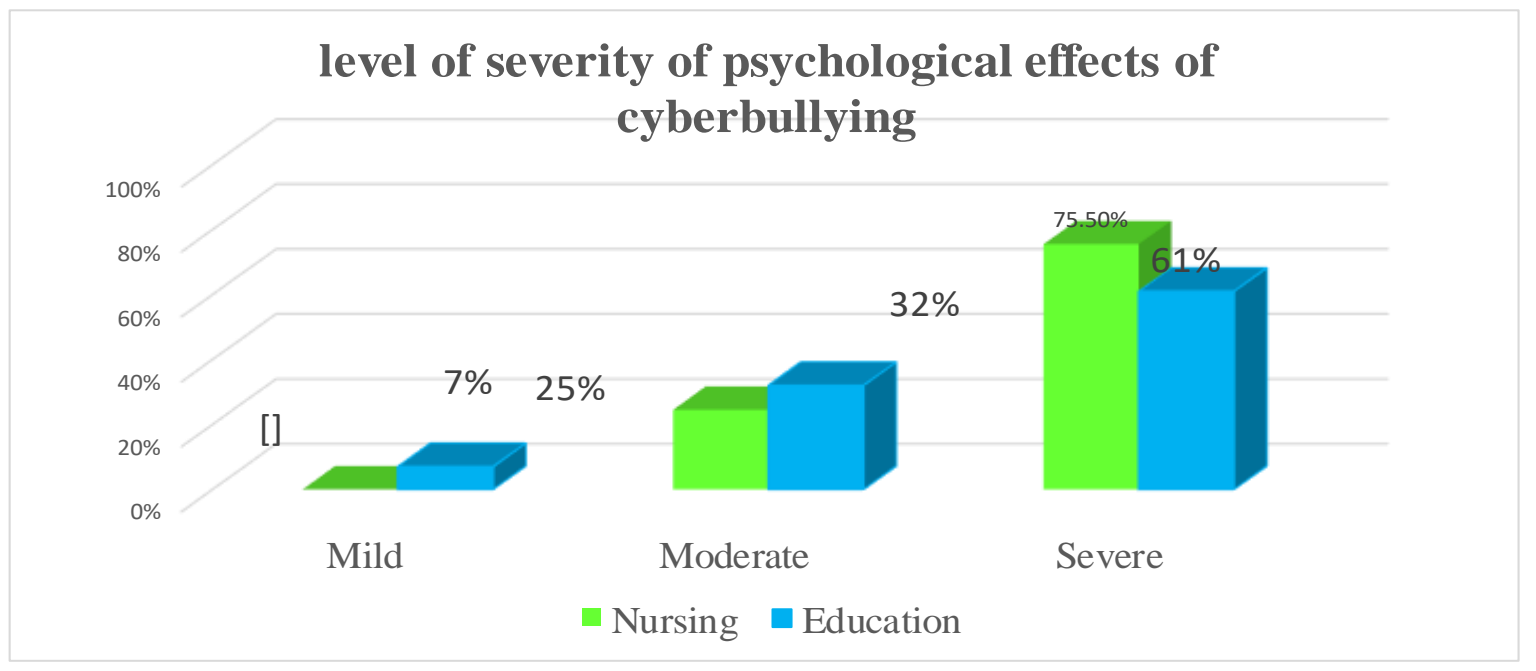

Figure (2): Percentage distribution of psychological effects of cyber bullying.

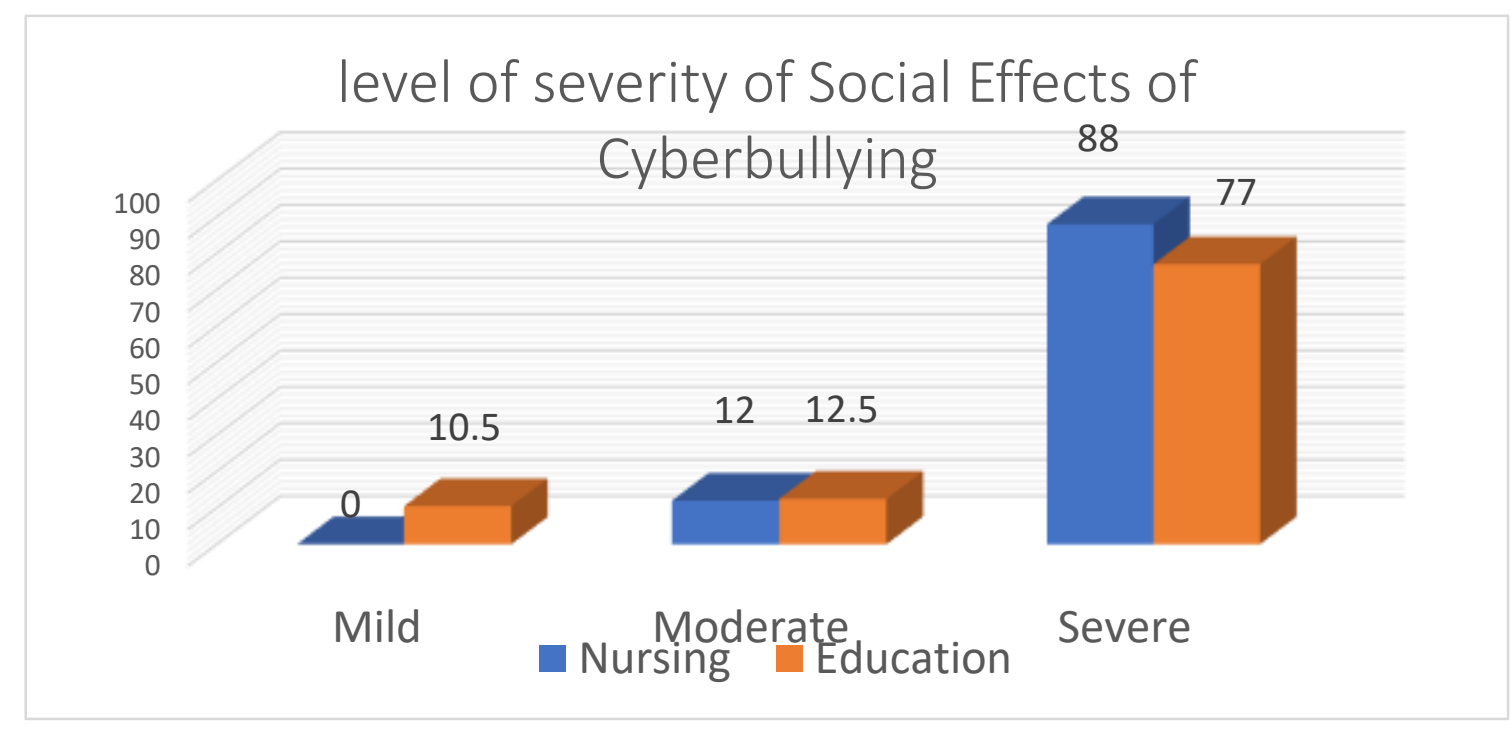

Figure (3): Percentage distribution of Social effects of Cyber bullying 
$\underline{\text { Ali Rezk El Khateeb, Mona Hassan Abdelal and Naglaa Fathi El-attar }}$

Table (2): Relation between sociodemographic characteristics and total knowledge of cyber bullying among studied students

\begin{tabular}{|c|c|c|c|c|c|c|c|c|c|c|c|c|}
\hline \multirow{4}{*}{$\begin{array}{c}\text { Sociodemogra } \\
\text { phic }\end{array}$} & \multicolumn{10}{|c|}{ Knowledge of cyber bullying } & \multirow{4}{*}{$\square^{2}$} & \multirow{4}{*}{$\begin{array}{c}\mathrm{P} \\
\text { valu } \\
\mathrm{e}\end{array}$} \\
\hline & \multicolumn{4}{|c|}{ Nursing students } & \multirow{3}{*}{$\square^{2}$} & \multirow{3}{*}{$\begin{array}{c}\mathrm{P} \\
\text { valu } \\
\mathrm{e}\end{array}$} & \multicolumn{4}{|c|}{ Education students } & & \\
\hline & \multicolumn{2}{|c|}{$\begin{array}{l}\text { Satisfacto } \\
\text { ry } \\
n=24\end{array}$} & \multicolumn{2}{|c|}{$\begin{array}{c}\text { Unsatisfact } \\
\text { ory } \\
n=176\end{array}$} & & & \multicolumn{2}{|c|}{$\begin{array}{c}\begin{array}{c}\text { Satisfactor } \\
\mathrm{y} \\
\mathrm{n}=46\end{array} \\
\end{array}$} & \multicolumn{2}{|c|}{$\begin{array}{c}\text { Unsatisfacto } \\
\text { ry } \\
n=154\end{array}$} & & \\
\hline & $\mathrm{N}$ & $\%$ & $\mathrm{~N}$ & $\%$ & & & $\overline{\mathrm{N}}$ & $\%$ & $\overline{\mathrm{N}}$ & $\%$ & & \\
\hline \multicolumn{13}{|l|}{ Age } \\
\hline $18<21$ & 0 & 0 & $\begin{array}{c}10 \\
4 \\
\end{array}$ & $\begin{array}{c}59.1 \\
\%\end{array}$ & \multirow{2}{*}{$\begin{array}{c}29.5 \\
45\end{array}$} & \multirow{2}{*}{$\begin{array}{c}0.00 \\
0\end{array}$} & 23 & $\begin{array}{c}50.0 \\
\%\end{array}$ & $\begin{array}{c}10 \\
7\end{array}$ & $69.5 \%$ & \multirow{2}{*}{$\begin{array}{c}5.90 \\
8\end{array}$} & \multirow{2}{*}{$\begin{array}{c}0.01 \\
5\end{array}$} \\
\hline $21<24$ & $\begin{array}{l}2 \\
4 \\
\end{array}$ & $\begin{array}{c}100 \\
\% \\
\end{array}$ & 72 & $\begin{array}{c}40.9 \\
\% \\
\end{array}$ & & & 23 & $\begin{array}{c}50.0 \\
\% \\
\end{array}$ & 47 & $30.5 \%$ & & \\
\hline \multicolumn{13}{|l|}{ Sex } \\
\hline Male & 0 & 0 & 65 & $\begin{array}{c}36.9 \\
\% \\
\end{array}$ & \multirow{2}{*}{$\begin{array}{c}13.1 \\
31\end{array}$} & \multirow{2}{*}{$\begin{array}{c}0.00 \\
0\end{array}$} & 26 & $\begin{array}{c}56.5 \\
\%\end{array}$ & 76 & $49.4 \%$ & \multirow{2}{*}{$\begin{array}{c}0.72 \\
9\end{array}$} & \multirow{2}{*}{$\begin{array}{c}0.39 \\
3\end{array}$} \\
\hline Female & $\begin{array}{l}2 \\
4 \\
\end{array}$ & $\begin{array}{c}100 \\
\% \\
\end{array}$ & $\begin{array}{c}11 \\
1 \\
\end{array}$ & $\begin{array}{c}63.1 \\
\% \\
\end{array}$ & & & 20 & $\begin{array}{c}43.5 \\
\% \\
\end{array}$ & 78 & $50.6 \%$ & & \\
\hline \multicolumn{13}{|l|}{ Marital status } \\
\hline Married & $\begin{array}{l}1 \\
6 \\
\end{array}$ & $\begin{array}{c}66.7 \\
\% \\
\end{array}$ & 26 & $\begin{array}{c}14.8 \\
\% \\
\end{array}$ & \multirow{2}{*}{$\begin{array}{c}34.2 \\
83\end{array}$} & \multirow{2}{*}{$\begin{array}{c}0 \\
.000\end{array}$} & 3 & $6.5 \%$ & 36 & $23.4 \%$ & \multirow{2}{*}{$\begin{array}{c}6.41 \\
0\end{array}$} & \multirow{2}{*}{$\begin{array}{c}0.01 \\
1\end{array}$} \\
\hline Single & 8 & $\begin{array}{c}33.3 \\
\%\end{array}$ & $\begin{array}{c}15 \\
0\end{array}$ & $\begin{array}{c}85.2 \\
\%\end{array}$ & & & 43 & $\begin{array}{c}93.5 \\
\%\end{array}$ & $\begin{array}{c}11 \\
8\end{array}$ & $76.6 \%$ & & \\
\hline \multicolumn{13}{|l|}{ Grade } \\
\hline First & 0 & 0 & 24 & $\begin{array}{c}13.6 \\
\%\end{array}$ & \multirow{4}{*}{$\begin{array}{c}45.4 \\
55\end{array}$} & \multirow{4}{*}{$\begin{array}{c}0.00 \\
0\end{array}$} & 9 & $\begin{array}{c}19.6 \\
\%\end{array}$ & 37 & $24.0 \%$ & \multirow{4}{*}{$\begin{array}{c}15.2 \\
75\end{array}$} & \multirow{4}{*}{$\begin{array}{c}0.00 \\
2\end{array}$} \\
\hline Second & 0 & 0 & 29 & $\begin{array}{c}16.5 \\
\%\end{array}$ & & & 17 & $\begin{array}{c}37.0 \\
\%\end{array}$ & 65 & $42.2 \%$ & & \\
\hline Third & 0 & 0 & 72 & $\begin{array}{c}40.9 \\
\% \\
\end{array}$ & & & 0 & 0 & 22 & $14.3 \%$ & & \\
\hline Fourth & $\begin{array}{l}2 \\
4\end{array}$ & $\begin{array}{c}100 \\
\%\end{array}$ & 51 & $\begin{array}{c}29.0 \\
\%\end{array}$ & & & 20 & $\begin{array}{c}43.5 \\
\%\end{array}$ & 30 & $19.5 \%$ & & \\
\hline
\end{tabular}


Table (3): Relation between sociodemographic characteristics and psychological effect of cyber bullying among studies students

\begin{tabular}{|c|c|c|c|c|c|c|c|c|c|c|c|c|c|c|c|c|}
\hline \multirow{4}{*}{ Items } & \multicolumn{14}{|c|}{ Psychological Effect of Cyber bullying } & \multirow{4}{*}{$\square^{2}$} & \multirow{4}{*}{$\begin{array}{c}\mathrm{P} \\
\text { value }\end{array}$} \\
\hline & \multicolumn{6}{|c|}{ Nursing students } & \multirow{3}{*}{$\square^{2}$} & \multirow{3}{*}{$\begin{array}{c}\mathrm{P} \\
\text { value }\end{array}$} & \multicolumn{6}{|c|}{ Education students } & & \\
\hline & \multicolumn{2}{|c|}{$\begin{array}{r}\text { Mild } \\
n=0\end{array}$} & \multicolumn{2}{|c|}{$\begin{array}{c}\text { Moderate } \\
n=49\end{array}$} & \multicolumn{2}{|c|}{$\begin{array}{l}\text { Severe } \\
\mathrm{n}=151\end{array}$} & & & \multicolumn{2}{|c|}{$\begin{array}{l}\text { Mild } \\
n=14\end{array}$} & \multicolumn{2}{|c|}{$\begin{array}{c}\text { Moderate } \\
n=64\end{array}$} & \multicolumn{2}{|c|}{$\begin{array}{l}\text { Severe } \\
\mathrm{n}=122\end{array}$} & & \\
\hline & $\mathrm{N}$ & $\%$ & $\overline{\mathrm{N}}$ & $\%$ & $\overline{\mathrm{N}}$ & $\%$ & & & $\mathrm{~N}$ & $\%$ & $\overline{\mathrm{N}}$ & $\%$ & $\mathrm{~N}$ & $\%$ & & \\
\hline \multicolumn{17}{|l|}{ Age } \\
\hline $18<21$ & 0 & 0 & $\begin{array}{l}2 \\
4\end{array}$ & $\begin{array}{c}49.0 \\
\%\end{array}$ & 80 & $\begin{array}{c}53.0 \\
\%\end{array}$ & \multirow{2}{*}{0.23} & \multirow{2}{*}{0.62} & $\begin{array}{l}1 \\
4\end{array}$ & 100.0 & $\begin{array}{l}3 \\
7\end{array}$ & $\begin{array}{c}57.8 \\
\%\end{array}$ & 79 & $\begin{array}{c}64.8 \\
o f\end{array}$ & \multirow[b]{2}{*}{8.99} & \multirow[b]{2}{*}{$0.01 *$} \\
\hline $21<24$ & 0 & 0 & $\begin{array}{l}2 \\
5\end{array}$ & $\begin{array}{c}51.0 \\
\%\end{array}$ & 71 & $\begin{array}{c}47.0 \\
\%\end{array}$ & & & 0 & 0 & $\begin{array}{l}2 \\
7\end{array}$ & $\begin{array}{c}42.2 \\
\%\end{array}$ & 43 & $\begin{array}{c}35.2 \\
\%\end{array}$ & & \\
\hline \multicolumn{17}{|l|}{ Sex } \\
\hline Male & 0 & 0 & $\begin{array}{l}1 \\
6\end{array}$ & $\begin{array}{c}32.7 \\
\%\end{array}$ & 49 & $\begin{array}{c}32.5 \\
\%\end{array}$ & \multirow{2}{*}{$\begin{array}{c}0.00 \\
1\end{array}$} & \multirow{2}{*}{0.97} & $\begin{array}{l}1 \\
4\end{array}$ & $\begin{array}{c}100.0 \\
\%\end{array}$ & $\begin{array}{l}3 \\
7\end{array}$ & $\begin{array}{c}57.8 \\
\%\end{array}$ & 51 & $\begin{array}{c}41.8 \\
\%\end{array}$ & \multirow{2}{*}{18.76} & \multirow{2}{*}{$\begin{array}{c}0.000 \\
* *\end{array}$} \\
\hline Female & 0 & 0 & $\begin{array}{l}3 \\
3\end{array}$ & $\begin{array}{c}67.3 \\
\%\end{array}$ & $\begin{array}{c}10 \\
2\end{array}$ & $\begin{array}{c}67.5 \\
\%\end{array}$ & & & 0 & 0 & $\begin{array}{l}2 \\
7\end{array}$ & $\begin{array}{c}42.2 \\
\%\end{array}$ & 71 & $\begin{array}{c}58.2 \\
\%\end{array}$ & & \\
\hline \multicolumn{17}{|c|}{ Marital status } \\
\hline $\begin{array}{l}\text { Marri } \\
\text { ed }\end{array}$ & 0 & 0 & $\begin{array}{l}1 \\
7\end{array}$ & $\begin{array}{c}34.7 \\
\%\end{array}$ & 25 & $\begin{array}{c}16.6 \\
\%\end{array}$ & \multirow{2}{*}{7.33} & \multirow{2}{*}{$\begin{array}{c}0.007 \\
*\end{array}$} & 0 & 0 & $\begin{array}{l}3 \\
0\end{array}$ & $\begin{array}{c}46.9 \\
\%\end{array}$ & 9 & $7.4 \%$ & \multirow{2}{*}{45.36} & \multirow{2}{*}{$\begin{array}{c}0.000 \\
* *\end{array}$} \\
\hline Single & 0 & 0 & $\begin{array}{l}3 \\
2\end{array}$ & $\begin{array}{c}65.3 \\
\%\end{array}$ & $\begin{array}{c}12 \\
6\end{array}$ & $\begin{array}{c}83.4 \\
\%\end{array}$ & & & $\begin{array}{l}1 \\
4\end{array}$ & $\begin{array}{c}100.0 \\
\%\end{array}$ & $\begin{array}{l}3 \\
4\end{array}$ & $\begin{array}{c}53.1 \\
\%\end{array}$ & $\begin{array}{c}11 \\
3\end{array}$ & $\begin{array}{c}92.6 \\
\%\end{array}$ & & \\
\hline \multicolumn{17}{|l|}{ Grade } \\
\hline First & 0 & 0 & $\begin{array}{l}1 \\
6\end{array}$ & $\begin{array}{c}32.7 \\
\%\end{array}$ & 8 & $5.3 \%$ & \multirow{4}{*}{$\begin{array}{c}32.8 \\
1\end{array}$} & \multirow{4}{*}{$\begin{array}{c}0.000 \\
* *\end{array}$} & 3 & $21.4 \%$ & $\begin{array}{l}3 \\
7\end{array}$ & $\begin{array}{c}57.8 \\
\%\end{array}$ & 6 & $4.9 \%$ & & \\
\hline Second & 0 & 0 & 0 & 0 & 29 & $\begin{array}{c}19.2 \\
\%\end{array}$ & & & $\begin{array}{l}1 \\
1 \\
\end{array}$ & $78.6 \%$ & 0 & 0 & 71 & $\begin{array}{c}58.2 \\
\%\end{array}$ & 105.2 & 0.000 \\
\hline Third & 0 & 0 & $\begin{array}{l}1 \\
6\end{array}$ & $\begin{array}{c}32.7 \\
\%\end{array}$ & 56 & $\begin{array}{c}37.1 \\
\%\end{array}$ & & & 0 & 0 & 3 & $4.7 \%$ & 19 & $\begin{array}{c}15.6 \\
\%\end{array}$ & 8 & $* *$ \\
\hline Fourth & 0 & 0 & $\begin{array}{l}1 \\
7\end{array}$ & $\begin{array}{c}34.7 \\
\%\end{array}$ & 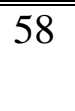 & $\begin{array}{c}38.4 \\
\%\end{array}$ & & & 0 & 0 & $\begin{array}{l}2 \\
4\end{array}$ & $\begin{array}{c}37.5 \\
\%\end{array}$ & 26 & $\begin{array}{c}21.3 \\
\%\end{array}$ & & \\
\hline
\end{tabular}

Not significant at $\mathrm{P}>0.05 *$ significant at $\mathrm{P} \leq 0.05 * *$ Highly significant at $\mathrm{P} \leq 0.001$ 
$\underline{\text { Ali Rezk El Khateeb, Mona Hassan Abdelal and Naglaa Fathi El-attar }}$

Table (4): Relation between sociodemographic characteristics and social effect of cyber bullying among studies students

\begin{tabular}{|c|c|c|c|c|c|c|c|c|c|c|c|c|c|c|c|c|}
\hline \multirow{4}{*}{$\begin{array}{l}\text { Sociodemog } \\
\text { raphic }\end{array}$} & \multicolumn{14}{|c|}{ Social effects of cyber bullying } & \multirow{4}{*}{$\square^{2}$} & \multirow{4}{*}{$\begin{array}{c}\mathrm{P} \\
\text { value }\end{array}$} \\
\hline & \multicolumn{6}{|c|}{ Nursing students } & \multirow{3}{*}{$\square^{2}$} & \multirow{3}{*}{$\begin{array}{c}\mathrm{P} \\
\text { valu } \\
\mathrm{e}\end{array}$} & \multicolumn{6}{|c|}{ Education students } & & \\
\hline & \multicolumn{2}{|c|}{$\begin{array}{c}\text { Mil } \\
\mathrm{d} \\
\mathrm{n}=0\end{array}$} & \multicolumn{2}{|c|}{$\begin{array}{c}\text { Modera } \\
\text { te } \\
n=24\end{array}$} & \multicolumn{2}{|c|}{$\begin{array}{l}\text { Severe } \\
\mathrm{n}=176\end{array}$} & & & \multicolumn{2}{|c|}{$\begin{array}{l}\text { Mild } \\
n=21\end{array}$} & \multicolumn{2}{|c|}{$\begin{array}{c}\text { Modera } \\
\text { te } \\
n=25\end{array}$} & \multicolumn{2}{|c|}{$\begin{array}{l}\text { Severe } \\
n=154\end{array}$} & & \\
\hline & $\overline{\mathrm{N}}$ & $\%$ & $\mathrm{~N}$ & $\%$ & $\overline{\mathrm{N}}$ & $\%$ & & & $\overline{\mathrm{N}}$ & $\%$ & $\overline{\mathrm{N}}$ & $\%$ & $\overline{\mathrm{N}}$ & $\%$ & & \\
\hline \multicolumn{17}{|l|}{ Age } \\
\hline $18<21$ & 0 & 0 & $\begin{array}{l}1 \\
6\end{array}$ & $\begin{array}{l}66 . \\
7 \%\end{array}$ & $\begin{array}{l}8 \\
8\end{array}$ & $\begin{array}{l}50 . \\
0 \%\end{array}$ & \multirow{2}{*}{$\begin{array}{c}2.3 \\
5\end{array}$} & \multirow{2}{*}{0.12} & $\begin{array}{l}1 \\
4\end{array}$ & $\begin{array}{l}66 . \\
7 \%\end{array}$ & $\begin{array}{l}2 \\
2\end{array}$ & $\begin{array}{l}88 . \\
0 \%\end{array}$ & $\begin{array}{l}9 \\
4\end{array}$ & $\begin{array}{l}61 . \\
0 \%\end{array}$ & \multirow{2}{*}{$\begin{array}{c}6.9 \\
0\end{array}$} & \multirow{2}{*}{$\begin{array}{c}0.03 \\
*\end{array}$} \\
\hline $21<24$ & 0 & 0 & 8 & $\begin{array}{l}33 . \\
3 \%\end{array}$ & $\begin{array}{l}8 \\
8 \\
\end{array}$ & $\begin{array}{l}50 . \\
0 \%\end{array}$ & & & 7 & $\begin{array}{l}33 . \\
3 \%\end{array}$ & 3 & $\begin{array}{l}12 . \\
0 \%\end{array}$ & $\begin{array}{l}6 \\
0\end{array}$ & $\begin{array}{l}39 . \\
0 \%\end{array}$ & & \\
\hline \multicolumn{17}{|l|}{ Sex } \\
\hline Male & 0 & 0 & $\begin{array}{l}1 \\
6\end{array}$ & $\begin{array}{l}66 . \\
7 \%\end{array}$ & $\begin{array}{l}4 \\
9\end{array}$ & $\begin{array}{l}27 . \\
8 \%\end{array}$ & \multirow[b]{2}{*}{$\begin{array}{l}14 . \\
51\end{array}$} & \multirow[b]{2}{*}{$\begin{array}{l}0.00 \\
0 * *\end{array}$} & $\begin{array}{l}1 \\
4\end{array}$ & $\begin{array}{l}66 . \\
7 \%\end{array}$ & $\begin{array}{l}2 \\
2\end{array}$ & $\begin{array}{l}88 . \\
0 \%\end{array}$ & $\begin{array}{l}6 \\
6\end{array}$ & $\begin{array}{l}42 . \\
9 \%\end{array}$ & \multirow{2}{*}{$\begin{array}{l}19 . \\
84\end{array}$} & \multirow{2}{*}{$\begin{array}{l}0.00 \\
0 * *\end{array}$} \\
\hline Female & 0 & 0 & 8 & $\begin{array}{l}33 . \\
3 \%\end{array}$ & $\begin{array}{l}1 \\
2 \\
7\end{array}$ & $\begin{array}{l}72 . \\
2 \%\end{array}$ & & & 7 & $\begin{array}{l}33 . \\
3 \%\end{array}$ & 3 & $\begin{array}{l}12 . \\
0 \%\end{array}$ & $\begin{array}{l}8 \\
8\end{array}$ & $\begin{array}{l}57 . \\
1 \%\end{array}$ & & \\
\hline \multicolumn{17}{|c|}{ Marital status } \\
\hline Married & 0 & 0 & 0 & 0 & $\begin{array}{l}4 \\
2\end{array}$ & $\begin{array}{l}23 . \\
9 \%\end{array}$ & \multirow{2}{*}{$\begin{array}{c}7.2 \\
5\end{array}$} & \multirow{2}{*}{$\begin{array}{c}0.00 \\
7 *\end{array}$} & 3 & $\begin{array}{l}14 . \\
3 \%\end{array}$ & 6 & $\begin{array}{l}24 . \\
0 \%\end{array}$ & $\begin{array}{l}3 \\
0\end{array}$ & $\begin{array}{l}19 . \\
5 \%\end{array}$ & \multirow[b]{2}{*}{$\begin{array}{c}0.6 \\
8\end{array}$} & \multirow[b]{2}{*}{0.71} \\
\hline Single & 0 & 0 & $\begin{array}{l}2 \\
4\end{array}$ & $\begin{array}{c}100 \\
\%\end{array}$ & $\begin{array}{l}1 \\
3 \\
4\end{array}$ & $\begin{array}{l}76 . \\
1 \%\end{array}$ & & & $\begin{array}{l}1 \\
8\end{array}$ & $\begin{array}{l}85 . \\
7 \%\end{array}$ & $\begin{array}{l}1 \\
9\end{array}$ & $\begin{array}{l}76 . \\
0 \%\end{array}$ & $\begin{array}{l}1 \\
2 \\
4\end{array}$ & $\begin{array}{l}80 . \\
5 \%\end{array}$ & & \\
\hline \multicolumn{17}{|l|}{ Grade } \\
\hline First & 0 & 0 & 8 & $\begin{array}{l}33 . \\
3 \%\end{array}$ & $\begin{array}{l}1 \\
6\end{array}$ & $\begin{array}{c}9.1 \\
\%\end{array}$ & \multirow{4}{*}{$\begin{array}{l}31 . \\
65\end{array}$} & \multirow{4}{*}{$\begin{array}{l}0.00 \\
0 * *\end{array}$} & 3 & $\begin{array}{l}14 . \\
3 \%\end{array}$ & $\begin{array}{l}2 \\
2\end{array}$ & $\begin{array}{l}88 . \\
0 \%\end{array}$ & $\begin{array}{l}2 \\
1\end{array}$ & $\begin{array}{l}13 . \\
6 \%\end{array}$ & & \\
\hline Second & 0 & 0 & 0 & 0 & $\begin{array}{l}2 \\
9\end{array}$ & $\begin{array}{l}16 . \\
5 \%\end{array}$ & & & $\begin{array}{l}1 \\
1\end{array}$ & $\begin{array}{l}52 . \\
4 \%\end{array}$ & 3 & $\begin{array}{l}12 . \\
0 \%\end{array}$ & $\begin{array}{l}6 \\
8\end{array}$ & $\begin{array}{l}44 . \\
2 \%\end{array}$ & 72. & 0.00 \\
\hline Third & 0 & 0 & \begin{tabular}{l|}
1 \\
6
\end{tabular} & $\begin{array}{l}66 . \\
7 \%\end{array}$ & $\begin{array}{l}5 \\
6\end{array}$ & $\begin{array}{l}31 . \\
8 \%\end{array}$ & & & 0 & 0 & 0 & 0 & $\begin{array}{l}2 \\
2\end{array}$ & $\begin{array}{l}14 . \\
3 \%\end{array}$ & 58 & $0 * *$ \\
\hline Fourth & 0 & 0 & 0 & 0 & $\begin{array}{l}7 \\
5\end{array}$ & $\begin{array}{l}42 . \\
6 \%\end{array}$ & & & 7 & $\begin{array}{l}33 . \\
3 \%\end{array}$ & 0 & 0 & $\begin{array}{l}4 \\
3\end{array}$ & $\begin{array}{l}27 . \\
9 \%\end{array}$ & & \\
\hline
\end{tabular}

Not significant at $\mathrm{P}>0.05 *$ *significant at $\mathrm{P} \leq 0.05 * *$ Highly significant at $\mathrm{P} \leq 0.001$ 
Table (5) Correlation between total knowledge scores, psychosocial and social effects among studied education students.

\begin{tabular}{|l||c||c|}
\hline \multicolumn{1}{|c||}{ Item } & $\mathrm{R}$ & P value \\
\hline $\begin{array}{l}\text { Knowledge \& Psychological } \\
\text { Effects }\end{array}$ & $-0.196^{* *}$ & 0.005 \\
\hline $\begin{array}{l}\text { Psychological Effects \& Social } \\
\text { Effects }\end{array}$ & $0.440^{* *}$ & 0.000 \\
\hline Knowledge \& Social Effects & $-0.548^{* *}$ & 0.000 \\
\hline
\end{tabular}

Table (6) Correlation between total knowledge scores, psychosocial and social effects among studied nursing students.

\begin{tabular}{|l||c||c|}
\hline \multicolumn{1}{|c||}{ Item } & $\mathrm{R}$ & P value \\
\hline $\begin{array}{l}\text { Knowledge \& Psychological } \\
\text { Effects }\end{array}$ & $-0.469^{* *}$ & 0.000 \\
\hline $\begin{array}{l}\text { Psychological Effects \& Social } \\
\text { Effects }\end{array}$ & $0.543^{* *}$ & 0.000 \\
\hline Knowledge \& Social Effects & $-0.608^{* *}$ & 0.000 \\
\hline
\end{tabular}

\section{Discussion:}

The results of the study revealed that more than half of Nursing students and less than two thirds of Faculty of Education students aged between (18-21) years old. In contrast, more than two thirds are females at Faculty of Nursing and less than half are females at Faculty of Education. Also, more than three quarters are single in both faculties respectively. These results were consistent with study of Dennehy et al (2020), which reported that majority of study sample was young single female.

\section{Regarding relation between} knowledge of cyber bullying, psychological, social effects of cyber bullying and sociodemographic characteristics of the current study results showed that there was highly statistically significant relation between sociodemographic characteristics and knowledge about cyber bullying among students at faculty of nursing. Also, there were statistically significant relation between age, marital status, studying year and knowledge about cyber bullying among students at faculty of education. It may be due to that if students' parents were informed they will know about cyber bullying causes, effects and how to help their children to deal with. And students with low level of knowledge about cyber bullying were more liable to be cyber victim and experience more psychological effects.

These results were consistent with study done by Bennett and Ramos (2019), which found that adolescents commonly participate in cyber bullying behavior as both perpetrators and victims. They found that, the number of cyber bullying cases and effects increased with age, lake of knowledge and cyber bullies were more often the perpetrators of cyber bullying. 


\section{$\underline{\text { Ali Rezk El Khateeb, Mona Hassan Abdelal and Naglaa Fathi El-attar }}$}

Also these results were consistent with study of Herrera-López et al (2017), which reported that sociodemographic characteristics like age, marital status and knowledge about cyber bullying had relation with psychological and social effect of cyber bullying.

This study clarified that there was highly statistically significant relation between number of siblings, order between siblings and awareness of cyber bullying. Beside highly statistically significant relation between studying level and psychological effects of cyber bullying among students. According to investigator's opinion small family had more care from parents and the more awareness about cyber bullying the more they know how to deal with.

This result was consistent with study of Cagirkan\& Bilek (2021), which reported that number of siblings was another important factor influencing the level of cyber bullying. The students with no siblings were more vulnerable to cyber bullying compared to those who have 2, 3 and more siblings. In addition, duration of mobile phone ownership affects the severity of cyber victimization.

These results also supported by study of Martín-Criado et al (2021), as they found that there was strong relation between cyber bullying perpetration knowledge of students and their parents about cyber bulling. And parents have a great involvement in cyber bullying behaviors. They cleared that families with high awareness about cyber bullying were in low risk for cyber bullying.

These results were on the same line with study of Llorent et al (2021), who reported that there was relation between studying level and cyber bullying victimization as they found that cyber bullying levels increases in adolescents in high schools and university. While they reported that more frequent bullying and cyberbullying involvement were found in Poland students in comparison

with Spain students.

These results were on the contrary with the study of Pandey and Sonker(2021), as they found that there was a negative correlation between cyber bullying behaviors and age of students. And a very low positive relation between cyber bullying behaviors and family type and number of siblings among this family. Also cleared that there was very low positive correlation between family income and cyber bullying behaviors.

As for study results showed that there was highly statistically significant relation between number of siblings, birth order, working status, student income and social effects of cyber bullying among sample students. Also, it is clarified that there was highly statistically significant relation between sex, marital status, studying level and social effects of cyber bullying among sample students. About this point investigator see that female affected by cyber bullying more than male socially and psychologically especially in eastern societies that's because of culture of this society and low self-esteem and low social support for females. While males with high family income are less liable to cyber bullying behaviors.

These results were consistent with research of Smith (2015), which found a significant difference by sex, for certain questions, and that girls are generally more exposed to cyber bullying effects than boys. Results also were consistent with study of Baldry et al (2016), which indicated that, 
with regard to cyber victimization, boys and girls were equally likely to be involved, whereas with regard to cyber bullying boys were up to 3 times more likely to act as either as an only cyberbully or as a cyberbully/cyber victim. Age differences could explain part of these results, meaning that cyber bullying is an age related issue, rather than a gender difference, with older girls involved in cyber bullying.

Results were on the same line with study of Espelage et al (2018), which founded that male adolescents reported more face-to-face bullying victimization compared to their female counterparts, and that females who were victims of bullying showed significantly lower academic self-efficacy and more social problems. Also Pala et al (2021), study which reported that cyber bullying effects increases when adolescents have reduced perceived social support level from their environment.

These results were in consistent with study of QiQi et al (2018), which revealed that familial and parental characteristics were demonstrated to be associated with adolescents' involvement in cyberbullying. Parents' divorce, separation and widow, below-median family income, mother's low education level, and father's unemployment were associated with greater risks of cyberbullying and other types of victimization.

This study denoted that there was highly statistically significant relation between knowledge, psychological and social effects of cyber bullying. There was negative correlation between knowledge of cyber bullying and psychosocial effects. In contrast, there was positive correlation between psychological and social effects of cyber bullying. According to investigator point of view psychological effects and social effects attached and one of them may lead to the other. While awareness with problem help in dealing with it and decrease its harmful effects.

On the same line the study of Brailovskaia et al (2018), which denoted that there was a relation between knowledge regarding cyber bullying and psychosocial consequences associated with cyber bullying. As well as study done by Grunin and Cohen (2020), which cleared that there was a significant relationship between adolescents' perceptions of cyber bullying , sociodemographic and youth cyber bullying behaviors.

These results were on the same line with the study of Calvete et al (2021), which cleared that increasing level of awareness about cyber bullying and its harmful consequences helping in decrease cyber bullying prevalence and effect of cyber bullying on individuals and society. Also results were consistent with study of Matulewska and Gwiazdowicz (2021), which denoted that cyber bullying exposure and cyber bullying harmful impact increased with low level about cyber bullying and its associated problems.

These results were in consistent with study of Grifoni et al (2021), who reported that there was a significant relation between knowledge about cyber bullying and liability to experience cyber bullying behaviors. And significant relation between awareness about cyber bullying phenomena and consequences of cyber bullying victimization.

Also this result was in harmony with the study done by Collen and Onan (2021), 


\section{$\underline{\text { Ali Rezk El Khateeb, Mona Hassan Abdelal and Naglaa Fathi El-attar }}$}

which stated that the social support received by these students from their friends and family might be increasing their resilience in situations that necessitate struggle. Strong family relations and spending time with one's family were suggested to increase the resilience of university students against the effects of online bullying.

These results not consistent with study of Wheeler et al (2018), which claimed that, despite a dramatic increase in public awareness, the prevalence of cyber bullying was still one of the most pressing issues facing adolescents. Cyber bullying is a persistent public health concern that has a significant impact in the university setting. And study of Ghoch and Pramanick (2021), which reported that there is a strong relation between socio-economic status of communities and psychological effects of cyber bullying on adolescents was conducted.

These results were not consistent with study of Hazlyna et al (2021), which indicated that the students in university are aware about cyber-bullying on social media. Furthermore, majority of the female students know what cyberbullying means even though majority of the female students are addicted to the social media. However, majority of them did not tell or report about cyberbullying to anyone and ask for help.

These results were on the same line with the study of Myers and Cowie (2019), which demonstrated that there was significant relation between psychosocial effect of cyber bullying and lack of knowledge amongst educators and their students about the legal consequences of cyberbullying behavior across the educational lifespan.

\section{Conclusion:}

While smart phones and new technology have an important role in new learning systems and have a lot of time during our daily life, they have very harmful effects especially cyber bullying which associated with a lot of psychosocial problems such as depression, anxiety, stress, social isolation, loneliness and suicide. Based on this study results there is a lack of students awareness about psychosocial problems associated with cyber bullying. So, it is important to teach student about cyber bullying and its associated effects to prevent them. There is a highly statically correlation between knowledge about cyber bullying and psychosocial problems among studied students.

\section{Recommendations:}

- Conducting psychoeducational program for, parents, teachers and students about psychosocial problems associated with cyber bullying.

- Increase community awareness about negative effect of cyberbullying through social media and television.

- Conduct a complementary study using parents of adolescents to determine the extent to their knowledge of cyberbullying and the strategies they use to protect their children from the negative effects of cyberbullying.

- For generalization replication of this study is recommended for a larger study sample.

- Investigate different types of bullying that are unexplained due to cultural and social factors.

- Conducting preventive, counselling program at the community to combat cyber bullying. 


\section{References:}

Baldry, A., Farrington, D\& Sorrentino, A. (2016). Cyber bullying in youth: A pattern of disruptive behavior. Psicología Educativa. 22 . 19-26.

Bennett, D., \& Ramos, M. (2019). Cyber bullying in Romantic Relationships: Developmental Perspectives. In Cyber bullying in Schools, Workplaces, and Romantic Relationships (pp. 184-200). Routledge.

Brailovskaia, J., Teismann, T., \& Margraf, J. (2018). Cyber bullying, positive mental health and suicide ideation/behavior. Psychiatry research, 267, 240-242.

Cagirkan, B., \& Bilek, G. (2021). Cyber bullying among Turkish high school students. Scandinavian journal of psychology.

Calvetea, E., Cortazara,N., FernandezGonzaleza, L., Echezarragaa,A., Beranuyb,M \& Oruea,I.(2021). Effects of a Brief Preventive Intervention in Cyberbullying and Grooming in Adolescents. Psychosocial Intervention .30(2). 75-84.

Collen,H\& Onan,N.(2021).cyber bullying and well-being among university students: the role of resilience. International Journal of Caring Sciences.14(1).632-641.

Dennehy, R., Meaney, S., Walsh, K. A., Sinnott, C., Cronin, M., \& Arensman, E. (2020). Young people's conceptualizations of the nature of cyber bullying: A systematic review and synthesis of qualitative research. Aggression and violent behavior, 51, 101379.

Espelage, D., Hong, J., Merrin, G., Davis, J., Rose, C., \& Little, T. (2018). A longitudinal examination of homophobic name-calling in middle school: Bullying, traditional masculinity, and sexual harassment as predictors. Psychology of violence, 8(1), 57.

Ghoch,S\&Pramanick,S.(2021).assessing psychological effects of cyber bullyingon the adolescents of cosmopolitan city. Space and culture India.8(4).89-105.

Grifoni, P., D'Andrea, A., Ferri, F., Guzzo, T., Felicioni, M \&Vignoli,A.(2021). Against Cyberbullying Actions: An Italian Case Study. Sustainability. 13. 1-15.

Grunin,L.,Yu,G.,\&Cohen,S.(2020). The relationship between youth cyber bullying behaviors and their perceptions of parental emotional support. International Journal of bullying prevention.13.1-12.

$\begin{array}{lrr}\text { Hazlyna,N., Abd Wahab,A., } & \text { Miswari,F., } \\ \text { Zulkipli,N., } & \text { Ghazali,N\& } & \text { Abdul } \\ \text { Razak,S.(2021). } & \text { Awareness } & \text { about } \\ \text { Cyberbullying on Social Media among Female } & \\ \text { Students in a Malaysian Public University. } \\ \text { Turkish Journal of Computer and Mathematics } \\ \text { Education.12.(3). 1592-1601. }\end{array}$

Herrera-López, M., Casas, J., Romera, E., Ortega-Ruiz, R., \& Del Rey, R. (2017). Validation of the European cyber bullying intervention project questionnaire for Colombian Adolescents. Cyberpsychology, Behavior, and Social Networking, 20(2), 117 125.

Kumar, R. (2021). Detection of Cyber bullying using Machine Learning. Turkish Journal of Computer and Mathematics Education (TURCOMAT), 12(9), 656-661.

Kumari, K., \& Singh, J. (2021). Identification of cyber bullying on multi-modal social media posts using genetic algorithm. Transactions on Emerging Telecommunications Technologies, 32(2), 3907. 
Llorent, V., Diaz-Chaves,A., Zych, I., Twardowska-Staszek,E.,\&Marin-Lopez,I.(2

021). Bullying and Cyberbullying in Spain and Poland, and Their Relation to Social, Emotional and Moral Competencies. School Mental Health.7.1-13.

Lopez-Vizcaino,M.,Novoa,F., Carneiro, V\& Cacheda, F.(2021). Early detection of cyber bullying on social media networks. Future generation computer system.118.219-229.

Martín-Criado, J., Casas, J.,\& Ortega-Ruiz, R.(2021). Parental Supervision: Predictive Variables of Positive Involvement in Cyberbullying Prevention. Int. J. Environ. Res. Public Health. 18. 1-12.

Matulewska, A., \& Gwiazdowicz, D.(2021). "I Would Kill the Director and Teachers in the School" Cyberbullying of Hunters in Poland. Int J Semiot Law. 34. 985-1010.

Megan,A\& Vaillancourt,T.(2017). The role of health care providers in cyber bullying. The Canadian journal of psychiatry.62(6).364-367.

Myers,C\& Cowie,H.(2019). Cyber bullying across the lifespan of education: Issues and interventions from school to university. Int. J. Environ. Res. Public health . 16. 1217.2-14.

PALA, S., ÜNSAL, A., EMİRAL, G., DEMIRTAŞ, Z., Zencirci, S., TÖZÜN, M., \& ARSLANTAŞ, D. (2021). Evaluation of cyber bullying and multidimensional perceived social support levels in high school students of Turkey. Journal of Istanbul Faculty of Medicine, 84(1), 104-112.

Pandey, A., \& Sonker, S. (2021). A Study of the Relationship between Cyber Bullying Behaviour and SES of Secondary School Students. International Journal of Recent
Advances in Multidisciplinary Topics, 2(6), 265-267.

QiQia,C., Camilla,L., Yuhongc,Z., Anned,C., Linga,C.,\& Patricke,I.(2018). Family poly-victimization and cyberbullying among adolescents in a Chinese school sample. Child Abuse \& Neglect. 77. 180-187.

Raheem, A., \& Al-Khaldi, M. (2021). Information and communication technology and the knowledge society. PalArch's Journal of Archaeology of Egypt/Egyptology, 18(08), 85-91.

Smith, P. (2015). The nature of cyber bullying and what we can do about it. Journal of Research in Special Educational Needs, 15(3), 176-184.

Wheeler,J.,Ward,C\&Rebowe,D.(2018).

Bullying nd cyber bullying prevention in schools. Position statement national association of school nurses.

Zych, I., Baldry, A., \& Farrington, P. (2017). School bullying and cyber bullying: Prevalence, characteristics, outcomes, and prevention. Handbook of behavioral criminology, 113-138. 


\section{إدراك الطلاب بالمشاكل النفس اجتماعية المرتبطة بالتنمر الإكتروني \\ على رزف الخطيب ــ منى حسن عبد العال ـ نجلاء فتحى العطار}

تتامت ظاهرة التنمر الإلكتروني بين فئة المراهقين وهو الأمر الذي قد يؤول إلى انتشار العديد من المنصات التفاعلية عبر وسائل التواصل الاجتماعي. و التنمر الإلكتروني هو مصطلح يمبل في تعريفه نحو التتمر التقني الذي يُستعان فيه بالأجهزة الإلكترونية مثل الكمبيوتر المحمول، الهواتف الذكية، أو الأجهزة اللوحية مما بسهل من خلالها الدخول إلى العديد من واجهات الإتصال بالانترنت التي تتبح إرسال رسائل عبر البريد اللأكتروني، أو التو اصل من خلال الدردشات الاجتماعية، أو انشاء مدونات شخصية، وكذلك النشر أو إرسال صور او مقاطع مصوّرة محرجة. لذا هدفت هذه الدراسة الى تقييم وعى الطلاب بالمشكلات النفس- اجتماعية المرتبطة بالتتمر الإلكتروني. وقد أجريت هذه الدر اسة على ( · . ع) طالب من كليتى التمريض و التربية بجامعة بنها ( · . ب) طالب من كل كلية ، حيث كثفت النتائج إلى وجود علاقة سلبية ذات دلالة إحصائية بين المعرفة بالتنمر الإلكتروني والتأثثرات النفسية والاجتماعية المرتبطه بالتتمر الالكترونى، كما اوصت الدراسة بعمل حمله توعوية مجتمعية لزيادة وعى الطالب والاسرة والمجتمع بالتنمر الالكترونى والمشاكل المترتبه عليه وكذلك طرق مو اجهته. 